\title{
Cutting Edge of Atomic Force Microscopy (AFM) of the Cell: From Live Cell Imaging to High-resolution Structural Analysis of Cytoskeletal Actin Filaments
}

Jiro Usukura $^{1}$, Eiji Usukura ${ }^{1}$, Akihiro Narita $^{1}$, Akira Yagi $^{2}$, Nobuaki Sakai $^{2}$, Yoshitsugu Uekusa ${ }^{2}$, Yuka Imaoka $^{2}$ and Shuichi Ito ${ }^{2}$

1. Structural Biology Research Center, Nagoya University, Nagoya, Japan.

2. Microsystem Technology Department, Olympus Corporation, Hachioji, Tokyo, Japan.

Increasing in scanning speed and mechanical stability improved in AFM facilitated live cell imaging and high-resolution structural analysis of organelles in cells. AFM also is an important tool for observing cells in physiological or native condition at higher resolution than light microscope (LM), and therefore enable to observe endocytosis and movement of cortical actin [1]. Even in the samples fixed chemically, AFM described intracellular fine structures containing water at comparable resolution to electron microscopy (EM) [2][3]. In addition, it should be noted that images formed by scanning needle while touching the sample surface in AFM bring peculiar structural information different from those in LM and $\mathrm{EM}$, in which images are formed by scattering, absorption or interference of electron or photon. Meanwhile, it has been not fully understood how the cantilever detects movement of actin filaments in cytoplasm beneath the cell membrane in living cells. In practice, however, recent high-speed AFM described moving filaments beyond cell membrane, despite such detection mechanism by cantilever remained unknown. Here, we will show various movements of filaments (mostly actin) depending on their spatial assignment in living cells, and also high-resolution structures of actin filaments in unroofed and fixed cells.

More recently, AFM detected successfully movement of fine filaments layered in relatively deep inside of living cells through the cell membrane at complete contact mode. When cantilever was approached slowly and contacted onto the living cell, AFM begin to display the surface structure of the cell. We call this state as the surface mode conveniently, in which movement of filo podia, lameli-podia and endocytosis were captured (Fig. 1). When cantilever was pushed down further a little bit, movement of many fine filaments (probably actin filaments) was visualized (Fig.2). We call such measurement as a cytoskeleton mode in live cell imaging. In EM, three types of actin filaments had been recognized beneath the cell membrane, i.e. actin filaments firmly attached to the membrane, actin filaments loosely attached to the membrane and actin filaments forming stress fibers in cytoplasm. Fine filaments extending randomly were disposed over the stress fibers clearly. Therefore, such filaments appeared to be located between cell membrane and stress fibers. Probably, these filaments seem to correspond to the type of actin filaments loosely attached to the membrane seen in EM. However, it is very curious that actin filaments firmly attached to the membrane as well as clathrin coat were not detected from outside of cell membrane at contact mode, despite these organelles were located just beneath the cell membrane in AFM of unroofed cells. Anyway, actin filaments extending randomly showed higher motility than ones forming stress fibers (Fig. 2). In general, stress fibers terminate on the cytoplasmic surface of ventral membrane corresponding to the focal contact between cell membrane and matrix or substrate. Similar focal points were also found in cytoplasm, in which many actin filaments were concentrated in one point. Such bundled actin filaments showed low motility similar to the stress fiber when compared with actin filaments arranged randomly and individually.

In order to view actin filaments and organelle more in detail, apical cell membrane was unroofed mechanically with customized low power sonication. All actin filaments showed short periodicity with 
about 5 6 $\mathrm{nm}$ derived from G-actin linked in beads form, and sometimes long periodicity of helical characteristic (Fig. 3). Furthermore, the polarity of actin filaments was determined based on the shape of a periodic striation without any decoration or labeling. However, unroofing technique is effective only in culture cells, but not in tissues. We tried to solve this problem by cryosectioning. Because the cantilever could access all parts in cryosection of a tissue sample after the embedding medium (sucrose) has been replaced with phosphate-buffered saline or distilled water. The use of cryosectioning enabled the morphological analysis and immunocytochemistry of cells in tissues in atomic force microscopy (AFM). In retinal tissue, the AFM images showed disks stacked and enveloped by the cell membrane in rod photoreceptor outer segments at EM resolution. In addition, three-dimensional architecture of synaptic ribbons, and the surface of the post-synaptic membrane facing the active site were revealed, which were not apparent using thin-section EM. AFM could depict the molecular binding of anti-opsin antibodies conjugated to a secondary fluorescent antibody bound to the disk membrane. The specific localization of the anti-opsin binding sites was verified through correlation with immunofluorescence signals in AFM combined with confocal fluorescence microscope [4].

\section{References:}

[1] Zhang Y et al, Microscopy 66 (2017), p. 272.

[2] Usukura E et al, Sci. Rep. 6 (2016), p. 27472.

[3] Usukura E et al, Sci. Rep. 7 (2017), p. 6462.

[4] The authors acknowledge funding from a governmental grant, AMED-SENTAN

$17 \mathrm{hm} 0102003 \mathrm{~s} 0106$

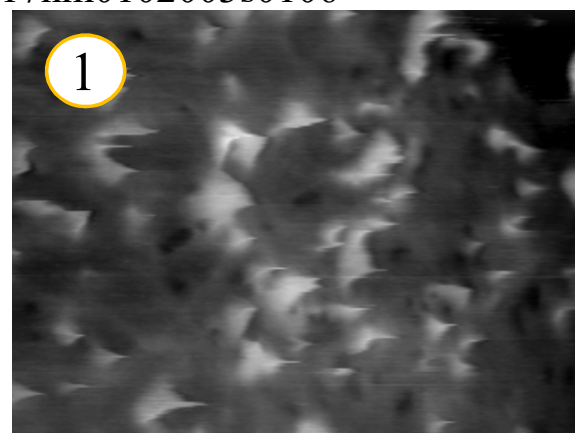

Figure 1. AFM image of living cell surface. Filo podia, lamelli-podia and several holes derived from endocytosis are found.

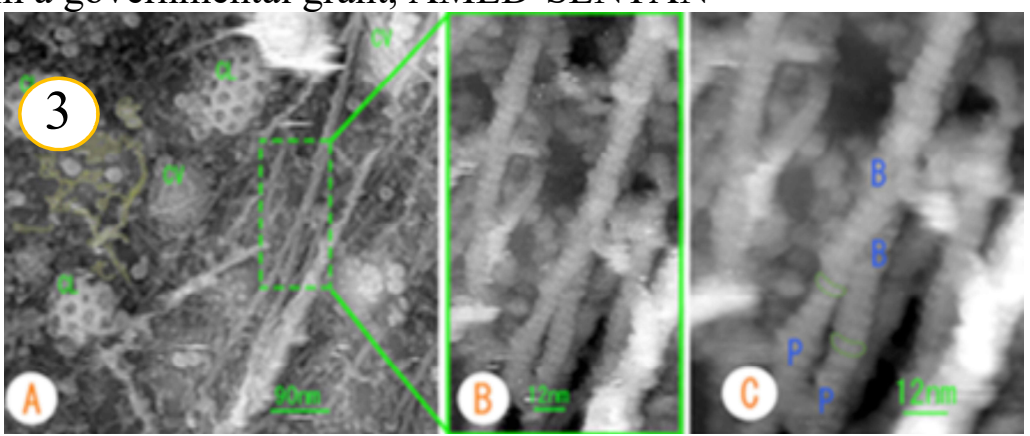

Figure 3. AFM images of cytoplasmic surface of cell membrane exposed by unroofing (A). B: High magnification image of boxed area. All actin filaments show short periodic striations. C: Averaged image of 10 consecutive scanning images of the same area in image $\mathrm{B}$

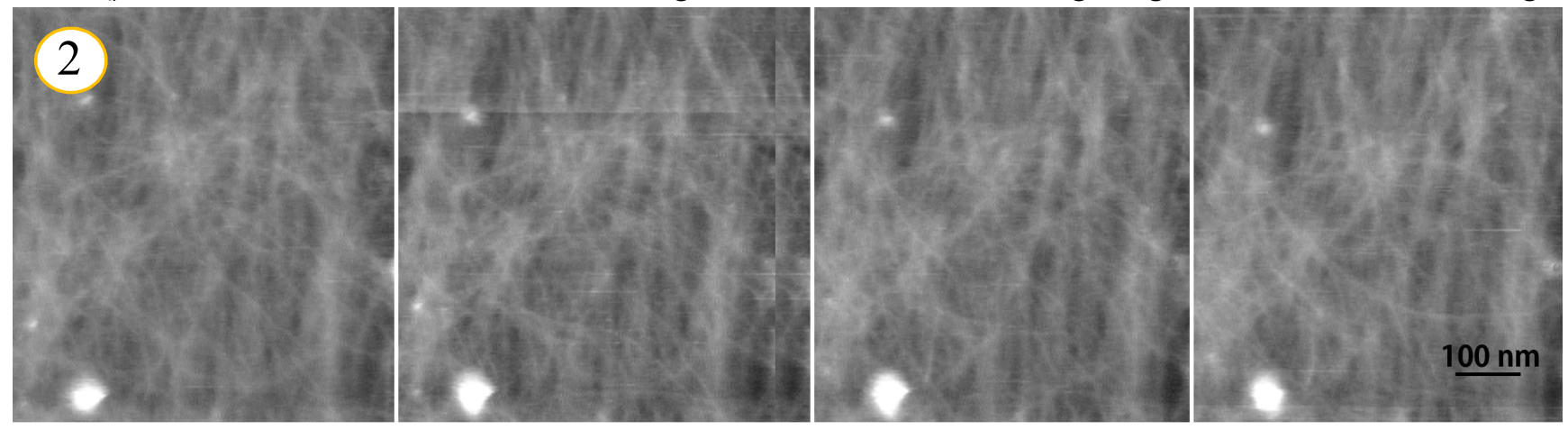

Figure 2. Consecutive images of a movie file showing movement of actin filaments. Actin filaments distributed randomly show higher motility comparing to the stress fibers located behind them. 